\title{
A Cutaneous Relapsing of a Diffuse Large B-Cell Lymphoma
}

\author{
Luciana Faria $^{1}$, Catarina Patronillo ${ }^{1}$, Sónia Fernandes ${ }^{1}$, and Cristina Gonçalves ${ }^{2}$ \\ ${ }^{1}$ Centro Hospitalar Póvoa de Varzim Vila do Conde EPE \\ ${ }^{2}$ Centro Hospitalar do Porto
}

September 25, 2021

\begin{abstract}
Diffuse large B cell lymphoma (DLBCL) it's the most frequent of all non-Hodgkin lymphomas. We describe a case of a man with multiple lesions on both legs being treated as venous ulcer for two months until a biopsy of them result on the diagnose of DLBCL.
\end{abstract}

\section{Introduction}

DLBCL represents the most common type of lymphoma. It is described as medium to large B cell neoplasm, with a diffuse growth pattern that may appear de novo or as a high-grade transformation from a small B-cell. The clinical presentation typically includes a fast-growing mass, B symptoms in one-third, and a median age of 70 years.

The secondary extra-nodal involvement was described in 25- $40 \%$ of cases, with skin been associated with a more aggressive course and poor prognosis. Therefore, skin biopsy should be performed promptly to identify the lymphoma and start on salvage chemotherapy.

This case represents a relapse of a DLBCL on skin that was treated for about two months as a benign lesions.

\section{Case report:}

\section{Patient Information}

In June 2018, a 69-year-old man with obesity, hypertension with hypertensive cardiopathy, presented to emergency with 3 weeks of evolution of anorexia, night sweats, asthenia and cervical edema. On physical examination multiple painless adenopathies were palpable at right cervical as so a spleen $4 \mathrm{~cm}$ below the costal margin. After cervical echography, a excisional biopsy was performed with diagnosis of a diffuse large B-cell lymphoma (DLBC), activated B-cell type. The completed studies shows stage IV-B (Ann Arbor staging system) and international prognostic index (IPI) 3. He was treated with half doses of Rituximabcyclophosphamide, doxorubicin, vincristine and prednisolone (R-CHOP), changing doxorubicin to mitoxantrone (R-CNOP) after $2^{\text {nd }}$ cycle due to cardiac toxicity. He did rituximab maintenance for two years. Two and half years later, he presented with two months of painless, indurated violaceus lesions on both low extremities, one of them with superficial ulcer (figure. 1). These was been treated has benign lesions of venous insufficiency.

\section{Diagnosis}

The biopsy of the lesion, by cytometry, confirms the relapse of DLBC. CT of chest, abdomen and pelvis with contrast revealed only intra-abdominal lymphadenopathy.

\section{Treatment}


He started on palliative chemotherapy with Bendamustine on a dose of the $120 \mathrm{mg} / \mathrm{m}^{2}$ by IV infusion on day 1 and 2 of a 28 -day cycle.

Follow up and Outcomes

The disease progress, with more lesions appeared in both arms and back. The patient dies after 4 months.

\section{Discussion}

Extranodal involvement of DLBCL is seen in $25-40 \%$ of patients and the most common extranodal sites are the gastrointestinal tract and skin. Cutaneous infiltration may occur through retrograde lymphatic spread, direct extension to the skin from adjacent lymph nodes or hematogenous dissemination. The presentations could be multiple and affect multiple areas, as in our case, with a combination of papules, nodules, plaques and a ulcer, affecting both legs. $(1,2)$

The outcome of relapsed of DLBCL is poor compared to the primary cutaneous diffuse B-cell lymphoma even more in presence of others unfavorable factors like high IPI score, age $>60$, reduced fitness and short time interval from previous chemotherapy. The median overall survival of 17 months, have been reported in those received any salvage treatment.(3)

Although these type of lesions could have a lot of differential diagnosis, in a patient with past history of DLBCL, the first attitude should be a skin biopsy detect lymphoma involvement and start treatment.

\section{References}

[1] Shamsudin N and Chang CC: Diffuse large B-cell lymphoma presenting with extensive cutaneous infiltration. Singap Med J 53(9):e198-200, 2012.

[2] Lee WJ, Won KH, Won CH, Chang SE, Choi JH, Moon KC, Park CS, Huh J, Suh C and Lee MW: Secondary cutaneous lymphoma: comparative clinical features and survival outcome analysis of 106 cases according to lymphoma cell lineage. British Journal of Dermatology, 173(1):134-145, 2015.

[3] Novo M, Castellino A, Nicolosi M, Santambrogio E, Vassallo F, Chiappella A, Vitolo U: High-grade B-cell lymphoma: how to diagnose and treat. Expert Rev Hematol:12(7):497-506, 2019. 


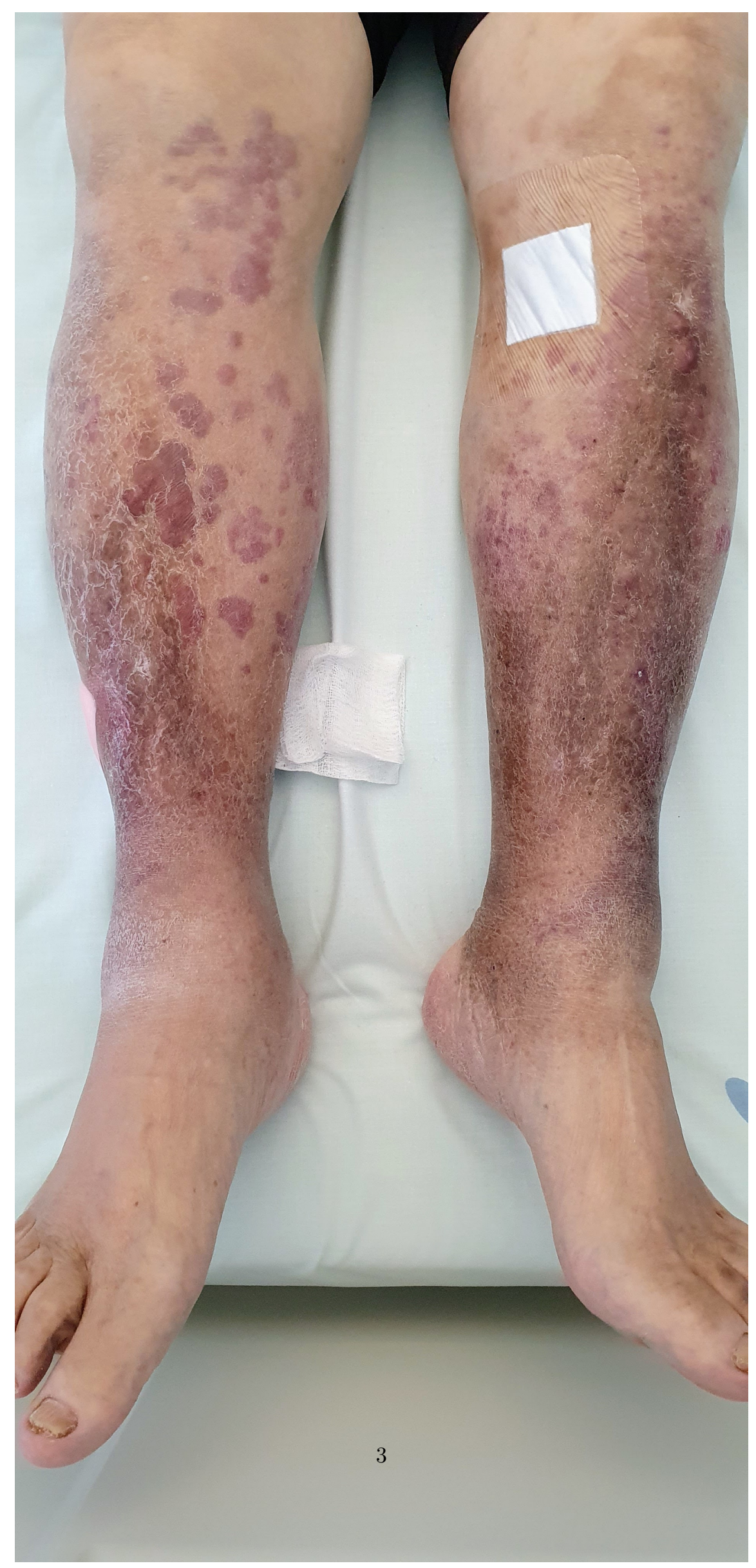

